\title{
Uso de APPs para la difusión de la gastronomía y establecimientos turísticos de alimentos y bebidas en la ciudad de Riobamba- Ecuador
}

\section{Use of APPs for the dissemination of gastronomy and tourist establishments of food and beverages in Riobamba city - Ecuador}

Susana Monserrat Zurita Polo. ${ }^{1}$, Paúl Eduardo Monge Moreno ${ }^{2}$, \& Nancy Maritza Montoya Ramírez. $^{3}$

\begin{abstract}
.
DOI https://doi.org/10.33262/cienciadigital.v3i4.2.1024

Riobamba city has a rich diversity of its cuisine due to the variety of spices and products in the country, its appeal is focused on the seasoning and creativity constantly renewed through the new generations of chefs and diners. In this area it is important to project to the world the gastronomic wonders that Riobamba city can offer, currently there are tools that facilitate this process in a global way reaching all corners of the world and this is thanks to the Internet and in a way more specific to "Social Networks" through the use of APPs. The dissemination of local gastronomy and tourist establishments of food and beverages through the Internet is leading to more and more people becoming direct and active users of Social Networks, who share culinary art as a common interest, leaving side limitations in terms of physical and temporal space, promoting their participation in information and knowledge empowerment activities. The present study allowed to identify the use of Social Networks for the dissemination of restaurant businesses in Riobamba city, a sample of 50 coffee shops, ice cream parlors, bars and restaurants was taken, the same ones that through their owners or administrators answered a survey on the use of PPPs and
\end{abstract}

\footnotetext{
${ }^{1}$ Escuela Superior Politécnica de Chimborazo, Facultad de Recursos Naturales. Riobamba, Ecuador, susana.zurita@espoch.edu.ec

${ }^{2}$ Unidad Educativa Santo Tomás Apóstol Riobamba. Riobamba, Ecuador. pmonge@uestar.edu.ec

${ }^{3}$ Instituto Superior Tecnológico Riobamba, Riobamba, Ecuador. nancymmr@gmail.com
} 
social networks for the dissemination of businesses, and a sample of 120 users of said tourist establishments, considered those who are 15 years old and older, determining that the most used social network in this regard is Facebook, through this platform they announce the services they offer: the menus and their gastronomy available to customers, as well as the creation of events, participation of activities, gastronomic marketing and general diffusion of the business.

Keywords: APPs, Social Networks, Gasronomy, Establishments of food and beverages

\section{Resumen.}

La ciudad de Riobamba en Ecuador, cuenta con una diversidad enriquecida de su gastronomía por la variedad de especias y productos existentes en el país, su atractivo se centra en la sazón y creatividad constantemente renovada a través de las nuevas generaciones de cocineros y comensales. Es importante proyectar al mundo las maravillas gastronómicas que Riobamba y la provincia de Chimborazo puede ofrecer, se cuenta con herramientas que facilitan este proceso de una forma global llegando a todos los rincones del mundo y esto es gracias a las "Redes Sociales" a través del uso de APPs. La difusión de la gastronomía chimboracense y establecimientos turísticos de alimentos y bebidas a través del Internet está dando lugar a que cada vez más personas se conviertan en usuarios directos y activos de las Redes Sociales, quienes comparten el arte culinario como un interés común, dejando de lado limitaciones en cuanto a espacio físico y temporal, promoviendo su participación en actividades de apoderamiento de información y conocimientos. El presente estudio permitió identificar el uso de Redes Sociales para la difusión de los negocios restauranteros en la ciudad de Riobamba, se tomó una muestra de 50 cafeterías, heladerías, bares y restaurantes, los mismos que a través de sus propietarios o administradores respondieron una encuesta sobre el uso de APPs y redes sociales para difusión de los negocios, y una muestra de 120 usuarios de dichos establecimientos turísticos, considerados aquellos que tienen edad de 15 años en adelante, determinando que la red social más utilizada en este sentido es Facebook, a través de esta plataforma dan a conocer los servicios que ofrecen: los menús y su gastronomía al alcance de los clientes, así como creación de eventos, participación de actividades, marketing gastronómico y difusión general del negocio.

Palabras claves: APPs, Redes sociales, Gastronomía, Establecimientos de alimentos y bebidas. 


\section{Introducción.}

En la actualidad el mundo está siendo sacudido por la velocidad para crear, producir y generar, ideas, información, conocimientos y saberes, que, apoyados en la ciencia y tecnología, hacen posible una difusión rápida y global. Las nuevas Tecnologías de la Información y de la Comunicación han evolucionado de manera vertiginosa en los últimos años, debido especialmente a su capacidad de interconexión a través de la Red, esta nueva fase de desarrollo tiene gran impacto en las organizaciones a todo nivel, sin dejar de lado las relacionadas con el ámbito gastronómico, la adaptación del entorno a este nuevo potencial y la adecuada utilización del mismo supone un reto sin precedentes.

Las innovaciones tecnológicas han proporcionado a la humanidad canales nuevos de comunicación e inmensas fuentes de información que difunden modelos de comportamiento social, actitudes, valores, formas de organización, etc. Estamos inmersos en la llamada sociedad de la información, hay que considerar entonces que los datos son la materia prima del siglo XXI.

La incorporación de las TIC y específicamente de las Redes Sociales a través de APPs en el ámbito gastronómico y establecimientos turísticos de alimentos y bebidas, permiten nuevas formas de acceder, generar, y transmitir información y conocimientos, promoviendo el ampliar las perspectivas de un negocio a través de una comunicación efectiva con nuevos clientes sin limitaciones de espacio ni tiempo ya sea de manera sincrónica y/o asincrónica.

Un objetivo clave para el funcionamiento de cualquier negocio es ofrecer un nivel más alto de lo normal en cuanto a servicios al cliente, más aún en el ámbito gastronómico donde el nivel de satisfacción del cliente puede ser reflejado de manera inmediata en un gesto como una sonrisa o por lo contrario una mueca como señal de desagrado, muchos de los negocios no tienen la oportunidad de crecer por la falta de difusión de sus servicios, los negocios entonces dependen de publicidad y la publicidad depende de las palabras; con la inclusión de los negocios en las Redes Sociales a través del Internet se visualiza como propietarios y administradores de establecimientos de alimentos y bebidas pretenden además de ofertarse, eliminar el distanciamiento con sus potenciales clientes a través de cartas digitales, promociones, eventos, etc.

La gastronomía es uno de los elementos más importantes en el turismo y que más movimiento genera en las redes sociales, en tal virtud en el presente artículo se tratarán aspectos a tomar en cuenta para elevar las potencialidades del uso de Internet, Redes sociales y APPs para la difusión de la Gastronomía de la provincia de Chimborazo y negocios relacionados al ámbito restaurantero. 


\section{Metodologia.}

El punto de partida para el presente trabajo es el uso de Internet, Sitios Web y APPs que permiten la comunicación directa con los usuarios esto es a través de las Redes Sociales. Además, se han utilizado encuestas que se han aplicado en varios restaurantes de la provincia de Chimborazo y han servido de base de comparación de oferta y difusión de servicios entre negocios que cuentan con sitios web o plataformas tecnológicas ya sea a través de videos o perfiles en las distintas Redes Sociales y los negocios que no.

Las técnicas utilizadas en el presente estudio son: observación directa, encuestas, entrevistas, a través de fuentes de información directas con los propietarios y administradores de los negocios, y fuentes secundarias y documentales por medio de revisión de bibliografía y sitios y páginas web referentes a la difusión de la gastronomía en Ecuador.

\section{Resultados.}

Las redes sociales se han convertido, en pocos años, en un fenómeno global, éstas se expanden como sistemas abiertos en constante construcción de sí mismos, al igual que las personas que las utilizan.

El término red, proviene del latín rete, hace mención a una estructura que tiene un determinado patrón; en informática una red es un conjunto de equipos interconectados que comparten información. (Pérez Porto \& Gardey, 2014)

En cuanto al término social, es relativo a la sociedad o conjunto de individuos que interactúan entre sí para formar una comunidad, pudiendo definir las Redes Sociales como estructuras en donde muchas personas mantienen algún tipo de vínculo ya sea amistad, comercial, laboral u otro.

Hoy en día el término "red social " se refiere a los diferentes sitios o páginas de internet que favorecen la creación de comunidades virtuales, ofreciendo a sus usuarios la posibilidad de contactarse con infinidad de individuos a fin de compartir contenidos, interactuar y crear comunidades sobre intereses similares: trabajo, lecturas, juegos, amistad, relaciones amorosas, entre otros. El término se atribuye a los antropólogos británicos Alfred RadcliffeBrown y Jhon Barnes.

Las redes sociales en Internet, engloban numerosas y variadas redes, por lo que generalmente, se habla de ellas en plural. Es difícil encontrar una persona que sólo posea una cuenta en uno de los servicios que ofrece Internet. Normalmente, los individuos son usuarios activos de varios servicios, mediante los cuales, crean y mantienen diferentes relaciones personales. Los servicios de redes sociales se están convirtiendo en un agregado de muchos servicios que funcionaban de forma separada. Se puede trasladar un estado de Twitter a Tuenti, o ver un 
vídeo en Youtube y compartirlo en Facebook o un estado en Whatsapp y/o Instagram. Todo esto integra a los usuarios de un modo complejo, por lo que el concepto de servicio de red social se diluye, y acaba siendo una combinación de posibilidades de comunicación.

Las redes sociales on-line se puede considerarlas del tipo horizontal a aquellas que no tienen una temática definida, están dirigidas a un público genérico, y se centran en los contactos, la motivación de los usuarios al acceder a ellas es la interrelación general, sin un propósito concreto, se centra en relacionar personas, comparten las mismas características: crear un perfil, compartir contenidos y generar listas de contactos, algunas son: Facebook, Instagram, MySpace, Orkut, Sonico, Tuenti, Netlog, Google+, Badoo, entre otras; y las Redes Sociales Verticales donde hay una tendencia hacia la especialización, estas pueden ser clasificadas por temática, redes tales como: Xing, LinkedIn, Viadeo; por actividad como Twitter, Muugoo, Plurk, Identi.ca,Tumblr, Wooxie, Second Life, World of Warcraft; o por contenido compartido como: Flickr, Fotolog, Youtube, Vimeo, SlideShare, Slideboom.

Las prácticas sociales se establecen mediante las actividades propuestas por el servicio que, habitualmente, son: Compartir contenido como fotografías, vídeos, páginas web, textos, música o noticias, enviar mensajes privados a otros usuarios, participar en juegos sociales que ofrece el servicio, comentar el contenido compartido por otros usuarios, publicar eventos para anunciar acontecimientos a su red de contactos, hablar en tiempo real con uno o más usuarios mediante chat o sistemas de conversión grupal, crear grupos exclusivos para determinados contactos, publicar comentarios en el perfil o espacio personal de otros usuarios.

La Red Social más utilizada a nivel mundial es Facebook, razón por la que el centro de atención en el presente estudio es esta red; creada por Mark Zuckerberg consistente en un sitio web de redes sociales, originalmente era un sitio para estudiantes de la Universidad de Harvard, pero actualmente está abierto a cualquier persona que tenga una cuenta de correo electrónico. Su infraestructura principal está formada por una red de más de 50.000 servidores que usan distribuciones del sistema operativo GNU/Linux usando LAMP. Ecuador ocupa la séptima posición en número de usuarios de Facebook entre los países de América del Sur, y la posición 34 en el ranking a nivel mundial.

En Ecuador según los resultados de la Encuesta de condiciones de vida realizada por el Instituto Nacional de Estadísticas y Censos (INEC, 2018), 4’224.984 habitantes que representan el 25,28\% de la población del Ecuador utilizan redes sociales esto reprde 5 años y más cuenta con cuenta con 6' 800.000 usuarios de los cuales el $47 \%$ son mujeres y $53 \%$ son hombres. Ecuador crece en Facebook a un promedio de 100.000 usuarios nuevos por mes. De acuerdo a las estadísticas de medición de Facebook las actividades de interés de los usuarios destacan las relacionadas con Comida y Restaurantes declaradas por 2’200.000 
usuarios de Facebook en Ecuador. El mayor porcentaje de usuarios en Facebook tiene edad entre 18 y 25 años, seguido muy de cerca por los de 26 a 35 años.

Según datos del Observatorio TIC, hasta el 2015, el $91 \%$ de personas que utilizan celular accedió a redes sociales, a través de su dispositivo móvil inteligente, es decir, 21 puntos porcentuales más que en el 2011, cuando se registró un $69,92 \%$ de personas que ingresaron a redes sociales desde sus teléfonos.

A nivel urbano se registra que el $92,4 \%$ de ciudadanos acceden a redes sociales, por medio de sus teléfonos móviles; mientras que en la zona rural el 82,88\% de personas con celular accede a las redes sociales.

Los datos, también evidencian que son los hombres los que más utilizan las redes sociales con un 91,38\%; en tanto que el $90,61 \%$ de las mujeres utilizan estas herramientas tecnológicas en sus teléfonos.

En cuanto a grupos etarios, las personas entre 15 y 29 años son las que más utilizan las redes sociales en sus teléfonos, con un 94,1\%; seguido de los jóvenes menores de 15 años que lo hacen en un 93\%. (Ministerio de Telecomunicaciones y de la Sociedad de la Información, 2019)

Las redes sociales utilizadas en Ecuador a enero de 2019 son: Facebook como la más popular con 12 millones de cuentas activas, registrando el $48 \%$ usuarias mujeres y $52 \%$ usuarios hombres; a continuación Instagram con 3,9 millones de usuarios de los cuales 55\% son mujeres y 45\% hombres; Linkedin 2,2 millones de usuarios, Snapchat 1,1 millones de cuentas activas y Twitter con 790.000 cuentas activas. El 80\% de usuarios se conectan desde dispositivos móviles, en los cuales descargan las APPs para su interacción con marcas y usuarios. (Zavala \& Sánchez, 2019)

Según la encuesta de condiciones de vida ECV 2014, la provincia de Chimborazo tiene una incidencia del $30,5 \%$ de uso de redes sociales con 4,78 horas promedio diarias de uso, a través de distintos medios como computadora con el $42,4 \%$ o teléfonos inteligentes con el 37,4\%, con acceso a internet $37,5 \%$ (INEC, 2018)

El uso de redes sociales para generación de interacciones proporciona oportunidades para pequeños y medianos negocios en el área de la gastronomía, generando comunicación y publicidad efectiva, relevante con su audiencia de clientes actuales y potenciales facilitando la creación de relaciones, solución de problemas y oferta de servicios y beneficios de calidad.

Según el Censo Económico realizado en el año 2010 en Ecuador existen 33.938 restaurantes, 8.217 panaderías, 8082 locales de comida rápida y 613 cafeterías; como dato adicional se puede mencionar que en este tipo de negocios restauranteros laboran 94.000 personas, de 
acuerdo a la encuesta nacional de Ingresos y Gastos de los hogares realizada en el año 2012, los ecuatorianos destinan en promedio \$585 millones en alimentos y bebidas no alcohólicas cada mes (Fuente INEC); la alimentación es una actividad vital, razón por la cual cada vez más ecuatorianos buscan una forma de emprender y obtener recursos económicos relacionados con esta actividad y maneras de promocionar su negocio a nivel global, optando por opciones de bajo costo y amplio alcance.

Una de las opciones más acogidas para la difusión de la gastronomía en la provincia de Chimborazo es el uso de redes sociales para lo cual se debe considerar varios aspectos como: la conversación es la base de una relación interpersonal, Twitter para hablar al minuto, Facebook para hablar cada día, Google Plus para hablar a la semana, Linkedin para hablar con profesionales, la seguridad en la red depende del sentido común de cada usuario, el factor humano es de gran importancia en Internet; las redes sociales pueden ser utilizadas como herramientas de comercialización a través de la llamada Realidad Aumentada y uso de nuevos dispositivos como teléfonos celulares inteligentes y tablets.

En la ciudad de Riobamba de acuerdo a las encuestas realizadas a una muestra de 50 cafeterías y negocios restauranteros, se determinó que el $85 \%$ de locales cuentan con al menos un perfil de negocio en redes sociales, publicaciones a través de videos en Youtube o uso de Whatsapp e Instagram para su promoción y difusión de los productos que ofrecen. Se determinó que el público al que va dirigido las publicaciones en mayor porcentaje está comprendido en un margen de edad de 15 a 35 años. Heladerías, cafeterías y bares realizan publicaciones para jóvenes de 15 a 25 años, mientras que los negocios restauranteros consideran como objetivo final adultos de 25 años en adelante.

El 80\% de los negocios restauranteros encuestados cuentan con páginas para su difusión principalmente en Facebook, el 20\% de los negocios han difundido sus servicios alguna vez a través de videos colocados en Youtube, el $42 \%$ están utilizando Whatsapp como medio de difusión de sus productos y para el público más joven a través de Instagram y Snapchat.

Es un estudio cuantitativo, de campo y descriptivo. La muestra estuvo conformada por 50 negocios restauranteros entre cafeterías, heladerías y restaurantes y 120 usuarios de diferentes edades, de los negocios restauranteros de la ciudad de Riobamba, provincia de Chimborazo. Se recolectó la información utilizando un cuestionario con 20 ítems con alternativas de respuesta valorada de la siguiente forma: siempre, frecuentemente, a veces, casi nunca, nunca. La escala mide tanto el grado de utilización de las redes sociales y su aceptación como el tipo de redes sociales utilizadas para la difusión y aceptación de los negocios restauranteros por parte del público en general.

El cuestionario fue realizado considerando aspectos básicos relacionados al uso de redes sociales: Tipo de Redes sociales, tiempo empleado para publicaciones, aceptación del 
público, elementos a difundir por medio de las redes sociales, medios digitales utilizados para la comunicación.

Cuadro 1. Redes sociales utilizadas por establecimientos turísticos de alimentos y bebidas en Riobamba

\begin{tabular}{lc}
\hline Red Social & Porcentaje \\
\hline Facebook & $80 \%$ \\
Whatsapp & $42 \%$ \\
Twiter & $20 \%$ \\
Instagram & $35 \%$ \\
Snapchat & $10 \%$ \\
Google + & $5 \%$ \\
Youtube & $20 \%$ \\
\hline
\end{tabular}

Fuente: Elaboración propia

Cuadro 2. Redes sociales utilizadas por usuarios de negocios restauranteros en Riobamba

\begin{tabular}{lc}
\hline Red Social & Porcentaje \\
\hline Facebook & $95 \%$ \\
Whatsapp & $90 \%$ \\
Twiter & $25 \%$ \\
Instagram & $50 \%$ \\
Snapchat & $30 \%$ \\
Google + & $10 \%$ \\
Youtube & $40 \%$ \\
\hline
\end{tabular}

Fuente: Elaboración propia 
Cuadro 3. Conocimiento de negocios restauranteros en Riobamba

\begin{tabular}{ll}
\cline { 2 - 2 } Medio de difusión & Porcentaje \\
\cline { 2 - 2 } Radio & $20 \%$ \\
Televisión & $25 \%$ \\
Redes sociales & $85 \%$ \\
Recomendación & $80 \%$ \\
Sitios Web & $28 \%$ \\
Volantes & $30 \%$ \\
\hline
\end{tabular}

Fuente: Elaboración propia

\section{Discusión.}

El uso de nuevas tecnologías en el ámbito gastronómico está revolucionando la forma de pensar tanto de propietarios y/o personas vinculadas al área como a los usuarios o clientes potenciales de negocios afines a la alimentación; cada vez es más frecuente y más sencillo obtener información sobre determinado restaurante o sobre alguna receta o profesional gastronómico en particular, la utilización de Redes Sociales está facilitando el acceso a la información sobre cocina ecuatoriana, tradicional, ancestral y de vanguardia, en fin a través de Redes como Youtube se está difundiendo de una manera atractiva lugares, tradiciones, platos, permitiendo a los usuarios ver a la Gastronomía Ecuatoriana con un panorama más amplio, existiendo un mejor aprovechamiento de recursos. (Marketing gastronómico, 2018)

Colocar la información en internet tiene como ventaja el acceso a la información desde cualquier ubicación, la información reside en servidores con conexión permanente; las herramientas Web 2.0 proporcionan mejoras en cuanto a interfaz, lo que posibilita una mejor y más fluida comunicación.

Entidades gubernamentales han visto en el uso de Internet una alternativa eficiente para la difusión de proyectos gastronómicos y alimentarios en Ecuador, tal es el caso de la difusión del Proyecto de Patrimonio Alimentario, el mismo que busca valorizar y proyectar la gastronomía nacional desde una mirada profunda que abarca desde sembríos hasta cuando los alimentos llegan a la mesa.

En la actualidad plataformas como Youtube son testigos de talento de jóvenes, empresarios, chefs, estudiantes y docentes quienes han visto en esta herramienta una opción para dar a 
conocer sus potencialidades en el campo gastronómico, es más habitual encontrar en la red fotos y videos de clases de cocina subidos para el deleite del público en general.

Las redes sociales como Facebook y Twiter se han convertido en espacios donde se puede mostrar al mundo fusiones de ingredientes, nuevas recetas, nuevos productos, facilitando una mejor oferta de los bienes y servicios brindados por los diversos restaurantes a nivel nacional; se da la oportunidad de integrar a Flashmobs para reuniones breves vía online con el propósito de obtener publicidad para movilizar a miles de personas; promueve el intercambio de experiencias con profesionales de diferentes áreas gastronómicas.

En Ecuador la Red Social más utilizada para difusión de la Gastronomía nacional, así como negocios restauranteros es Facebook, en esta red se han creado grupos de interés tales como Gardemanger, Asociación de Chefs del Ecuador, Foro Panamericano de Asociaciones Gastronómicas Profesionales, Academy of Culinary Professional of Americas, entre otros, en los cuales diariamente participan profesionales de la rama gastronómica. Le sigue de cerca Whatsapp según las estadísticas de redes sociales en Ecuador, Facebook es usada en un 97\%, frente al 90\% de uso de Whatsapp, 65\% de uso de Instagram, 49\% de uso de Twiter, 26\% de uso de la red Snapchat y en menores porcentajes otras redes sociales. (BRANDEC, 2018)

El compartir nuevos conocimientos como: técnicas vanguardistas, productos utilizados en las diferentes zonas y en la elaboración de platos autóctonos y de autor, así como expandir la gastronomía ecuatoriana internacionalmente, tener información actualizada acerca de temas de interés, además permiten acudir a eventos, participar en actos y conferencias tanto presenciales como virtuales y que pueden ser en tiempo real son ventajas del uso de las Redes sociales en el ámbito gastronómico.

Hay muchas dinámicas para producir contenido gastronómico en Internet, permite recolectar información sobre costumbres gastronómicas, restaurantes más visitados y lugares turísticos; los profesionales gastronómicos pueden dar uso a las redes sociales para masificar la educación y la cultura gastronómica ecuatoriana, por tanto, usuarios y proveedores de servicios deben trabajar en este camino en el que la educación y la concienciación de los riesgos que puede conllevar para la privacidad el hecho de no proteger adecuadamente los datos se erige como aspecto clave a la hora de exponer información en las redes sociales. (Revista Líderes, 2018)

Los restaurantes pueden dar a conocer los productos y servicios a miles de personas que hacen uso de las redes sociales ya que es una excelente forma de hacer mercadotecnia y publicidad a bajos costos. El consumidor puede interactuar y conocer las características de los productos, además de promociones, noticias de los establecimientos restauranteros y lanzamiento de nuevos productos; es una perfecta fuente de información para saber lo que quiere el cliente y en un futuro retomar la misma para los planes estratégicos del restaurante. 
Hay que considerar también que las Redes sociales pueden llegar a ser peligrosas en cuanto a si no se configura la privacidad correctamente, puede haber violación de los derechos de autor, así como pueden darse casos de suplantación de menús, de productos específicos de un restaurante. La excesiva información de productos elaborados dentro de los establecimientos restauranteros, ocasiona la pérdida de identidad del restaurante, porque pueden apoderarse de todos los contenidos publicados y además ser utilizados por profesionales poco éticos que pudieren aprovecharse de los recursos para beneficio propio perjudicando notablemente al autor. Adicionalmente se debe tomar muy en cuenta que los malos comentarios generados en la red pueden dar mala imagen al negocio y perjudicarlo.

\section{Conclusiones.}

- Cada vez es más frecuente el uso de internet y Redes Sociales en el ámbito gastronómico y empresarial a nivel provincial y nacional, por las múltiples ventajas que puede brindar tanto a dueños de restaurantes como a usuarios y consumidores.

- La gastronomía chimboracense y ecuatoriana puede ser conocida y apreciada a nivel mundial a través del Internet, entidades de gobierno como Ministerio de Turismo, así como negocios restauranteros nacionales se promueven a través de este medio.

- El Internet a través de uso de las APPs y Redes Sociales reduce el tiempo de comercialización y disminuye los costos de comunicación, por lo que se ha convertido en una herramienta de comercio valorado por las empresas y negocios en general incluidos los restauranteros a nivel nacional.

- La entrega de información de Internet en un formato digital a bajo costo ayuda a los negocios a funcionar con mayor eficiencia, ya que pueden pasar los ahorros para beneficio del cliente en cuanto a mejora de servicios por parte del restaurante en este caso.

- El uso de redes sociales implica tener conciencia de las ventajas que puede traer a un negocio en cuanto a publicidad y/o difusión de servicios, pero de la misma forma se deberá considerar el tipo de información que se coloca ya que ésta estará al alcance de todo el mundo, por lo que se deberá poner atención sobre todo en lo relacionado a seguridad y privacidad

\section{Referencias bibliográficas.}

BRANDEC. (2018). Hablemos de marcas. Recuperado el 20 de 03 de 2018, de Estadísticas de redes sociales en Ecuador 2017: http://www.hablemosdemarcas.com/estadisticasde-redes-sociales-en-ecuador-2017/

El emprendedor. (2017). Errores al usar Facebook en la empresa. Recuperado el 23 de 03 de 2018, de http://www.elemprendedor.ec/errores-al-usar-facebook-en-la-empresa/ 
INEC. (2018). ECUADOR EN CIFRAS. Obtenido de Estadísticas Sociales: http://www.ecuadorencifras.gob.ec/documentos/webinec/Estadisticas_Sociales/TIC/2016/170125.Presentacion_Tics_2016.pdf

Marketing gastronómico. (2018). Aprende a gestionar tu restaurant como un auténtico profesional. Obtenido de http://marketingastronomico.com/

Ministerio de Telecomunicaciones y de la Sociedad de la Información. (2019). Ministerio de Telecomunicaciones y de la Sociedad de la Información. Obtenido de https://www.telecomunicaciones.gob.ec/91-de-ecuatorianos-utiliza-las-redessociales-en-su-telefono-inteligente/

Pérez Porto, J., \& Gardey, A. (2014). Obtenido de Definición de:: https://definicion.de/redsocial/

Revista Líderes. (2018). Revista líderes. Recuperado el 20 de 03 de 2018, de El sabor nacional se convierte en una experiencia: https://www.revistalideres.ec/tag/gastronomia

Zavala, V., \& Sánchez, J. (2019). Global Digital Report. Obtenido de https://www.ekosnegocios.com/articulo/facebook-es-la-red-social-mas-utilizada-en-ecuador

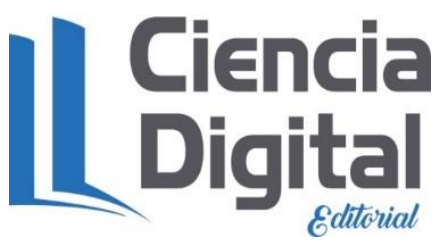




\section{PARA CITAR EL ARTÍCULO INDEXADO.}

Zurita Polo, S., Monge Moreno, P. E., \& Montoya Ramírez, N. M. (2019). Uso de APPs para la difusión de la gastronomía y establecimientos turísticos de alimentos y bebidas en la ciudad de RiobambaEcuador. Ciencia Digital, 3(4.2), 191-203. https://doi.org/10.33262/cienciadigital.v3i4.2.1024

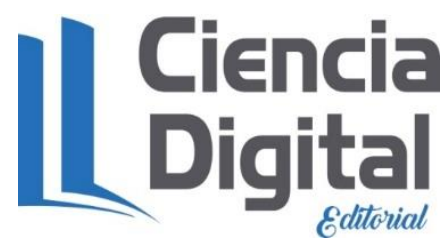

El artículo que se publica es de exclusiva responsabilidad de los autores y no necesariamente reflejan el pensamiento de la Revista Ciencia Digital.

El articulo queda en propiedad de la revista y, por tanto, su publicación parcial y/o total en otro medio tiene que ser autorizado por el director o editor de la Revista Ciencia Digital. 